\title{
Zur Gleichstellung beim Entgelt von Frauen und Männern mit gleicher Qualifikation
}

\author{
Lohnungleichheit ist ein zentrales Element, das einer Gleichstellung von Frauen und Männern \\ entgegensteht. Selbst bei Ausschluss bekannter lohnrelevanter Unterschiede, d. h. bei gleicher \\ Qualifikation, besteht nach wie vor eine Lohnlücke zulasten der Frauen: der bereinigte Gender \\ Pay Gap. Geburtsbedingte Mehrkosten für Unternehmen könnten einen Teil des bereinigten \\ Gender Pay Gap erklären. Zur Kompensation dieser Mehrkosten und zur Beendigung einer \\ Diskriminierung in der Lohnfindung werden mögliche Ansatzpunkte diskutiert.
}

Gleichstellung oder Gleichbehandlung von Frauen und Männern ist eine komplexe Aufgabe. Die wissenschaftliche Literatur dazu ist immens, politische Absichtserklärungen sind Legion, der Auftrag des Grundgesetzes ist von apodiktischer Klarheit. Vieles davon ist in Deutschland in die bisherigen Gleichstellungs- und Familienberichte der Bundesregierung sowie in die Veröffentlichungen des dafür zuständigen Bundesministeriums für Familie, Senioren, Frauen und Jugend (BMFSFJ, 2020) eingeflossen. Die EUKommission (2021) hat kürzlich einen Vorschlag für eine Richtlinie mit dem Ziel einer stärkeren Anwendung des Prinzips des gleichen Lohns für gleiche Arbeit oder Arbeit von gleichem Wert zwischen Männern und Frauen insbesondere über eine umfassende Entgelttransparenz vorgelegt. Schon diese Überblicksveröffentlichungen zeigen die Fülle der qualitativen und quantitativen Dimension der Problematik. Sie fokussieren sich vor allem auf die Ungleichheit zwischen Männern und Frauen in Erwerbstätigkeit und Sorgearbeit sowie beim Entgelt oder bei der Rente. Ein besonderes Augenmerk gilt derzeit den kurz- und längerfristigen Auswirkungen der Corona-Pandemie auf die Ungleichheit (Kohlrausch und Zucco, 2020; Zucco und Lott, 2021).

Die Frage der Gleichbehandlung hat wechselwirkend eine familiäre und eine unternehmensbezogene Dimension. Gibt es eine Vorabentscheidung, wer von beiden Partnern mehr Haushalts- und Familienarbeit übernimmt, folgt oft daraus, wer seine Arbeit im Unternehmen für längere Zeit einschränken oder unterbrechen muss. Gibt es Unterschiede im Arbeitseinkommen und sind diese entschei-

(C) Der/die Autor:in(nen) 2021. Open Access: Dieser Artikel wird unter der Creative Commons Namensnennung 4.0 International Lizenz veröffentlicht (creativecommons.org/licenses/by/4.0/deed.de).

Open Access wird durch die ZBW - Leibniz-Informationszentrum Wirtschaft gefördert.

* Der Autor dankt Angela Nottelmann für hilfreiche Kommentare bei der Entstehung dieses Textes. dungsbestimmend, folgen daraus Unterschiede im Maße der Haushalts- und Familienarbeit. ${ }^{1}$ Gleichbehandlung muss sich deshalb zugleich am Arbeitsplatz und im privaten Haushalt verwirklichen. Diese Forderung wird besonders dann virulent, wenn es den Wunsch nach Kindern gibt und diese zu betreuen sind. Gleiches gilt im Falle der Betreuung von alten und kranken Familienangehörigen.

Gleich- und Ungleichbehandlung haben darüber hinaus Auswirkungen auf einzel- und gesamtwirtschaftliche Produktivität und Wettbewerbsfähigkeit. Wenn im Unternehmen zu große Lohnunterschiede bestehen - so die EUKommission (2021) -, „könnte dies zu Unzufriedenheit (und in der Folge zu geringerer Produktivität) und daraus resultierenden immateriellen Kosten führen." (EU-Kommission, 2021, 7) Umgekehrt gilt: „Aus der Sicherung von Arbeitsplätzen, der Bindung von Arbeitskräften und mehr Produktivität ... wird sich der Vorschlag positiv auf die Rentabilität der Unternehmen und das Funktionieren des Binnenmarkts auswirken." (EU-Kommission, 2021, 6) Auf dieser makroökonomischen Ebene sieht die Kommission dann höhere Gesamtbruttoverdienste, dadurch eine Zunahme der Gesamtnachfrage sowie der Staatseinnahmen aus direkten Steuern und Sozialbeiträgen (EU-Kommission, 2021, 10).

1 Der Begriff der Familie steht hier stellvertretend für andere Formen der Partnerschaft.

Dr. Willi Koll war Ministerialdirigent in den Grundsatzabteilungen des Bundeswirtschafts- und des Bundesfinanzministeriums sowie Mitglied im Wirtschaftspolitischen Ausschuss der EU und der OECD sowie im Makroökonomischen Dialog der EU. 


\section{Die aktuelle Situation}

Die Realität ist noch weit von der zuvor skizzierten Norm entfernt. Speziell ist der durchschnittliche Lohn für Frauen erheblich niedriger als der für Männer. Vor allem aber gibt es auch bei sonst gleichen Bedingungen einen Unterschied in der Bezahlung von Frauen gegenüber Männern. Messgröße ist hier die unbereinigte Lohnlücke (Unbereinigter Gender Pay Gap; GPG). Sie entsteht als Ergebnis vielfältiger Einflussgrößen. Das sind vor allem die Wahl von Beruf und Branche, der Führungs- und Qualifikationsanspruch, der Beschäftigungsumfang sowie Bildung und Berufserfahrung. Will man die unbereinigte Lohnlücke verkleinern, ist an all diesen Stellschrauben anzusetzen (BMFSFJ, 2020, 9).

Es verbleibt jedoch ein Rest des GPG, der nach dem Einfluss von Beruf und Branche den größten Beitrag zum GPG ausmacht, die sogenannte bereinigte Lohnlücke (Bereinigter Gender Pay Gap; BGPG) (BMFSFJ, 2020, 9). Auch wenn es gelingen sollte, alle bekannten Einflussgrößen - Tätigkeiten und Qualifikationen (Destatis, 2021, a) - zwischen Frau und Mann anzugleichen, verbleibt ein Unterschied. ${ }^{2}$ Eine Klärung hat auch das Entgelttransparenzgesetz in seiner bisherigen Fassung nicht erbracht.

Eine wesentliche Voraussetzung zur Verifizierung dieser „sonst gleichen Bedingungen“ sind diskriminierungsfreie Arbeitsbewertungsverfahren. Einige Autor:innen sehen diese nicht als gegeben an und identifizieren einen nichtneutralen Arbeitsbewertungseffekt zulasten der Frauen (Klammer, Klenner und Lillemeier, 2018). Die EU-Kommission hat in ihrem Richtlinienvorschlag die Ableitung von Kriterien für eine von jeder Diskriminierung freie vergleichende Arbeitsbewertung als ein zentrales Element erkannt. Deshalb enthält ihr Vorschlag eine ausführliche Behandlung dieser Frage (EU-Kommission, 2021).

Für Deutschland wird - jeweils in Bruttostundenverdiensten - für 2020 eine unbereinigte Lohnlücke von $18 \%$ (2006: $23 \%$ ) und für 2018 eine bereinigte Lohnlücke von $6 \%$ (2006: $8 \%$ ) genannt (Destatis, 2021, a). Deutschland hat damit eine der höchsten unbereinigten Lohnlücken in der EU (14\%) (Schmieder und Wrohlich, 2021). Dagegen fällt die bereinigte Lohnlücke im internationalen Vergleich relativ gering aus (Institut der deutschen Wirtschaft, 2021, 5). Für die EU beträgt sie zwei Drittel des Unbereinigten GPG von $14 \%$, also rund $9 \%$ (EU-Kommission, 2021, 1). Die unbereinigte Lohnlücke klafft zwischen West-

2 "Da solche Verzerrungen oft nicht wahrgenommen werden, kann Lohntransparenz das Problembewusstsein bei den Arbeitgebern schärfen und ihnen dabei helfen, diskriminierende geschlechtsspezifische Lohnunterschiede, die nicht auf gerechtfertigten Entscheidungen im Rahmen ihres Ermessensspielraums beruhen und häufig nicht beabsichtigt sind, zu erkennen." Europäische Kommission (2021, 1 f.). und Ostdeutschland mit $18 \%$ bzw. $6 \%$ (2020) stark auseinander; ${ }^{3}$ dagegen fällt die bereinigte Lohnlücke in beiden Landesteilen mit $6 \%$ bzw. $7 \%$ (2018) annähernd gleich hoch aus (Destatis, 2021, a). Wenn man die Jahre 2018 und 2020 vergleicht, ist ein Drittel des unbereinigten GPG für Westdeutschland unerklärt. Für Ostdeutschland dürfte das für die gesamte unbereinigte Lohnlücke gelten.

Andere Untersuchungen kommen zu abweichenden Ergebnissen. Schmidt (2016) ermittelt einen BGPG von 3,8\%, Boll und Leppin (2015) errechnen eine Bandbreite von 2,3\% bis $8,3 \%$. Klammer, Klenner und Lillemeier $(2018,59)$ nennen nach der Bereinigung des GPG von 21,3\% (2018) um den Effekt von Merkmalsunterschieden von 11 Prozentpunkten eine bereinigte Größe von $10 \% .{ }^{4}$ Das Institut der deutschen Wirtschaft $(2021,5)$ errechnet eine Bandbreite von $2 \%$ bis $7 \%$. Angesichts dieser Größenordnung und weil er mehr oder weniger alle beschäftigten Frauen betrifft, lohnt es sich, den Gründen für den BGPG nachzugehen. ${ }^{5}$

Eine erste Vermutung geht dahin, den BGPG quasi residual einer Diskriminierung zuzuschreiben. Als Ausdruck von Diskriminierung werden unter anderem Vorlieben (taste discrimination), systematischer Ausschluss von Frauen von Männerjobs und Selbstausschluss („deliberate decision by women not to invest into skills demanded by those discriminating firms") (Akyol, Neugart und Pichler, $2015,7)$ oder - als mittelbare Diskriminierung - die oben genannte nichtneutrale Arbeitsbewertung zulasten der Frauen genannt. Eine völlige Zuschreibung des BGPG als Diskriminierung wird jedoch infrage gestellt: „Der unerklärte Teil der Lohnlücke darf jedoch aus mehreren Gründen nicht mit Diskriminierung gleichgesetzt werden" (Boll und Leppin, 2015). Derartige Gründe sind hier „Unterschiede in unbeobachteten Jobattributen oder Personalmerkmalen“, „Verfahren der Arbeitsbewertung“ sowie „unterschiedliche Verhaltensweisen von Männern und Frauen in Lohnverhandlungen“.

Andere Stimmen fokussieren sich auf monetäre Effekte. „Denn vielleicht fehlen einkommensrelevante Merkmale in der Berechnung, die gar nicht diskriminierend sind. Ihr Beitrag zur Erklärung der Entgeltdifferenzen wäre dann verdeckt im unerklärten Anteil enthalten.“ (BMFSFJ, 2020,

3 Diese Diskrepanz verdient eine eigene Untersuchung. Ein Grund hierfür dürfte in der unterschiedlichen Sozialisation vor der Wiedervereinigung zu suchen sein.

4 "Die Differenz zwischen dem von uns errechneten Wert und dem vom Statistischen Bundesamt ausgewiesenen „bereinigten“ Gender Pay Gap lässt Rückschlüsse auf die Rolle einer nicht geschlechtsneutralen Arbeitsbewertung zu“ (Klammer, Klenner und Lillemeier, 2018, 64).

5 Ein BGPG von $6 \%$ wächst sich über einen längeren Zeitraum auf eine beträchtliche Größenordnung aus. Bei z.B. einem Jahreslohn von 40.000 Euro und einer Lebensarbeitszeit von 40 Jahren beträgt er fast 100.000 Euro (BMFSFJ, 2020, 13). 
10). Anders ausgedrückt: „Vor allem ist es schwierig, diskriminierende Entgeltungleichheit von einer marktbedingten Entgeltungleichheit zu unterscheiden." (BMFSFJ, 2020, 11). Das Statistische Bundesamt kommentiert hierzu: „Bei der Interpretation des bereinigten Gender Pay Gap ist zu beachten, dass hierbei nur in der Statistik vorliegende Informationen Berücksichtigung finden. Der Wert des bereinigten Gender Pay Gap kann somit als eine Obergrenze interpretiert werden. Es ist jedoch davon auszugehen, dass die Unterschiede geringer ausfielen, wenn weitere Informationen über lohnrelevante Einflussfaktoren für die Analysen zur Verfügung stünden, vor allem Angaben zu Erwerbsunterbrechungen." (Destatis, 2020, a. Zu untersuchen ist also, ob es zusätzliche „einkommensrelevante Merkmale" oder eine „marktbedingte Entgeltungleichheit" gibt, die bisher unerklärt geblieben sind.

\section{Der Unternehmenssektor}

Der Vermutung einer marktbedingten und einkommensrelevanten Entgeltungleichheit wird hier mit dem Fokus auf die Nachfrage nach Arbeitskräften nachgegangen. Sie verweist auf den Unternehmensbereich und insbesondere auf den Rentabilitätskalkül des Unternehmens. Sucht man nach möglichen Ursachen für die lohnbezogene Ungleichbehandlung in Form des BGPG unter sonst gleichen Bedingungen, steht als primärer Unterschied zwischen Mann und Frau im Vordergrund, dass die Mutterschaft unabdingbar mit einer Unterbrechung der Berufstätigkeit verbunden ist. Die zentrale Frage ist dann, ob diese Unterbrechung ein wesentlicher oder sogar ausschlaggebender Grund für diese Ungleichbehandlung durch Unternehmen ist. Eine Untersuchung zu Auswirkungen der Unterbrechung der Erwerbstätigkeit aufgrund der Geburt und Erziehung von Kindern konstatiert hier: „Daher bleibt methodisch nur die Möglichkeit, die Mutterschaft über einen Abzug bei der Berechnung der potentiellen Berufserfahrung zu berücksichtigen" (Finke, Dumpert und Beck, 2017, 58). Im Ergebnis führt dieser Ansatz ,... unter den dort gemachten Annahmen zu einem um rund 0,2 bis 0,3 Prozentpunkte niedrigeren bereinigten Gender Pay Gap.“

Es bleibt die Frage, ob es nur diese Art der Berücksichtigung von Mutterschaft im Unternehmensbereich mit dem eher marginalen Ergebnis gibt. Zu untersuchen ist daher, ob und inwieweit durch Mutterschaft die Rentabilität eines Unternehmens nicht anderweitig als durch den Unterschied in der Berufserfahrung betroffen ist.

Auswirkungen von geburtsbedingter Abwesenheit auf die Unternehmensrentabilität

Bei gleicher Qualifikation, wie im BGPG unterstellt, werden die Arbeitsleistung, gemessen an der Produktivität pro Stunde, und damit auch der Kosten- und Gewinnbeitrag von Männern und Frauen - hier vor der Schwangerschaft und nach der Rückkehr in das Unternehmen - als gleich hoch angenommen. Frauen und Männer verdienen deshalb, den gleichen Stundenlohn zu erhalten. Dagegen bedeutet eine geburtsbedingte Abwesenheit der Mutter in einer rein betriebswirtschaftlich begrenzten Betrachtung

- qualitativ eine Unterbrechung in den entsprechenden internen Unternehmensbereichen, aber auch in der Beziehung zu den Kund:innen, wo es besonders auf kontinuierliche persönliche Bindungen und Verbindungen ankommen kann.

- quantitativ einen Rückgang der Produktivität während der Schwangerschaft, eine Lücke in Produktion oder Dienstleistung während der geburtsbedingten Abwesenheit sowie eine Phase, in der nach Wiederbeschäftigung gegebenenfalls verlorene Qualifikation oder Produktivität aufgeholt werden muss. Darüber hinaus gehen viele bisherige Vollzeitkräfte nach der Geburt inres ersten Kindes in Teilzeit.

Je kleiner dabei das Unternehmen, umso größer ist der relative Anteil einer solchen Unterbrechung am Betriebsgeschehen. Ein Unternehmen könnte auf die Erwartung dieser Unterbrechung, soweit allein Rentabilität die Entscheidung bestimmt und die Diskontinuität als gravierend eingeschätzt wird, bei seiner Nachfrage nach Arbeitskräften reagieren $^{6}$, indem es bei der Einstellung Männer tendenziell bevorzugt, Frauen für gleiche Arbeit weniger bezahlt, sie mit Arbeit betraut, bei der die Kosten einer geburtsbedingten Abwesenheit geringer sind, und sie bei der Beförderung und der Besetzung von Führungspositionen benachteiligt.

\section{Geburtsbedingte Mehrkosten}

Über die Lebensarbeitszeit gesehen kann eine Frau in Beschäftigung einmal oder mehrfach schwanger werden, Mutterschutz nehmen und darüber hinaus zur Betreuung des Kindes eine Zeit lang zuhause bleiben. Für die Zeit des Mutterschutzes erhält die Frau Mutterschaftsgeld von max. 13 Euro pro Tag von der Krankenversicherung. Das Unternehmen stockt diesen Betrag auf den zeitanteiligen durchschnittlichen Nettolohn auf. Diese Aufstockung wird dem Unternehmen aus der Umlage U2 erstattet. Nicht ausgeglichen werden dagegen etwaige Mehrkosten wegen schwangerschaftsbedingter Minderbelastbarkeit und geburtsbedingter Abwesenheit der Mutter:?

6 Eine solche Verhaltensweise würde dann auch Frauen betreffen, die von vornherein keine Kinder haben möchten.

7 Der Wiedereintritt in das Unternehmen wird als problemlos angenommen, solange die Abwesenheit von begrenzter Dauer ist. 
- Minderbelastbarkeit: Von der Dauer der Schwangerschaft von 40 Wochen werden bei einem guten Verlauf bis zum Mutterschaftsurlaub 34 Wochen im Unternehmen verbracht. Während dieser Zeit nimmt die Belastbarkeit zunehmend ab.

- Überstundenarbeit: Soweit die Übertragbarkeit der entfallenden Arbeit auf das vorhandene Personal möglich ist, fallen Einarbeitung und Überstundenzuschläge an. Ihre Höhe variiert zwischen $15 \%$ und $40 \%$; bei Schichtdienst geht sie darüber hinaus. ${ }^{8}$

- Schwangerschaftsvertretung: Wenn eine volle oder zeitweise Vertretung möglich ist, dürfte es hier zu Einstellungs- und Einarbeitungskosten sowie einer eventuell geringeren (qualitativen und quantitativen) Produktivität der Ersatzarbeitskräfte kommen.

- Fehlender Ersatz: In manchen Bereichen ist ein Ersatz von vornherein nicht vorgesehen. Bei Vollbeschäftigung oder Arbeits- und Fachkräftemangel ist eine Übertragung der Arbeit der Abwesenden auf eine Ersatzarbeitskraft gar nicht erst möglich. Infolgedessen fallen Produktion, Umsatz und Gewinn aus und damit Opportunitätskosten in Höhe des entgangenen Gewinns an.

Soweit derartige Mehrkosten entstehen, können sie als Prozentsatz $(k)$ eines Bruttostundenverdiensts eines männlichen Mitarbeiters (w) ausgedrückt werden. Eine Abnahme der Belastbarkeit während der Zeit der Schwangerschaft im Unternehmen wird hier rechnerisch als Abwesenheit in einem Anteil dieser Zeit ausgedrückt $\left(t_{a}\right)$; sie und die anschließende Zeit der Abwesenheit $\left(t_{b}\right)$ addieren sich zur gesamten rechnerischen Abwesenheitszeit $\left(t=t_{a}\right.$ $+t_{b}$, in Stunden). Die Mehrkosten über die gesamte Abwesenheitszeit betragen dann:

$\mathrm{K}=\mathrm{k} \cdot \mathrm{w} \cdot \mathrm{t}[$ Euro $]$

Die Mehrkosten pro Stunde, bezogen auf die Gesamtstundenzahl der Lebensarbeitszeit $(\mathrm{T})$, betragen dann:

$\mathrm{K} / \mathrm{T}=\mathrm{k} \cdot \mathrm{w} \cdot \mathrm{t} / \mathrm{T}[$ Euro/h]

Bezogen auf den Bruttostundenverdienst eines männlichen Mitarbeiters (w) ergibt sich folgender Mehrkostensatz:

$k^{\prime}=(K / T) / w=(k \cdot w \cdot t / T) / w=k \cdot t / T[\%]$

8 Vgl. Arbeitsvertrag.org (2021).
Bei einer durchschnittlichen Kinderzahl pro Frau $(x)$ gilt dann:

$k^{\circ}=k^{\prime} \cdot x=k \cdot t / T \cdot x[\%]$

Der BGPG ist seinerseits als eine durchschnittliche Lohnlücke ebenfalls für jedes Jahr der Lebensarbeitszeit als Prozentsatz eines Bruttostundenverdiensts eines männlichen Mitarbeiters definiert. Insofern lässt sich ein auf die Lebensarbeitszeit verteilter Mehrkostensatz, der gleichermaßen auf einen männlichen Bruttostundenverdienst bezogen ist, als ein Anteil am BGPG beschreiben:

$B G P G(k):=k^{\circ} / B G P G=(k \cdot t / T \cdot x) / B G P G[\%]^{9}$

Der Mehrkostensatz ( $k$ ) lässt sich ableiten aus dem Aufschlagsatz für Überstunden $\left(k_{1}\right)$, Mehrkosten und Minderproduktivität der Schwangerschaftsvertretung $\left(k_{2}\right)$ und entgangenem Gewinn $\left(k_{3}\right)$, jeweils bezogen auf den oben genannten Bruttostundenverdienst und multipliziert mit ihrer jeweiligen Dauer $\left(\mathrm{t}_{\mathrm{i}}\right)$ :

$k=k_{1} \cdot t_{1}+k_{2} \cdot t_{2}+k_{3} \cdot t_{3} \quad$ mit $t_{1}+t_{2}+t_{3}=t$

Zur Veranschaulichung wird ein Zahlenbeispiel abgeleitet. Der BGPG ist ein Durchschnittswert über die Gesamtwirtschaft, deshalb werden hier ebenfalls gesamtwirtschaftliche Durchschnittsgrößen zugrunde gelegt. Ferner wird angenommen, dass der Kostensatz für Überstunden, Schwangerschaftsersatz und entgangenem Gewinn als prozentualer Aufschlag auf den Bruttoverdienst gleich hoch ist. Mit $\mathrm{k}=40 \%{ }^{10}, \mathrm{t}=1,3 \mathrm{Jahre}^{11}, \mathrm{x}=1,5^{12}$ Kinder

9 Dieser abgeleitete Anteil am BGPG kann als jährliche Quasi-Umlage über die gesamte Lebensarbeitszeit zulasten des Lohnes von Frauen zum Ausgleich geburtsbedingter Mehrkosten interpretiert werden.

10 Als durchschnittlicher Überstundensatz werden $40 \%$ angenommen (IG Metall, 2018). Mehrkosten für Ersatz können nicht beziffert werden. Der Satz für entgangenen Gewinn wird hier gewählt als gesamtwirtschaftliches Verhältnis von Betriebsüberschuss/Selbständigeneinkommen, d.h. ohne Vermögenseinkommen, zu Bruttolöhnen und -gehältern; es lag 2019 bei $41 \%$. Destatis (2021, b), eigene Rechnung.

11 Der Mutterschutz beträgt 14 Wochen. Für die Minderbelastbarkeit während einer unterstellten Zeit der Schwangerschaft im Unternehmen von 34 Wochen wird ein Abwesenheitsäquivalent von knapp einem Drittel, d.h. rund zehn Wochen und von der Geburt bis zum Besuch einer Kita ein Zeitraum von einem Jahr zugrunde gelegt. Die rechnerische geburtsbedingte Abwesenheitsdauer würde sich dann insgesamt auf 68 Wochen oder 1,3 Jahre pro Kind belaufen.

12 Die durchschnittliche Kinderzahl pro Frau liegt in Deutschland seit 2015 bei 1,5. (Destatis, 2020, b). Bei 1,5 Kindern pro Frau beträgt dann die gesamte Abwesenheit 1,5 · 1,3 Jahre = 1,95 Jahre. Eine Publikation des BMFSFJ (2010) nennt hierfür Beispiele aus der Praxis: „Frauen in nichtakademischen Medizinberufen (Frauenanteil $=90,1 \%$ ) haben durchschnittlich 1,84 Jahre geburtsbedingte Auszeiten, bei der Untergruppe von Frauen mit tatsächlichen Auszeiten sind es durchschnittlich sogar 2,67 Jahre. Zum Vergleich: Die Werte für Frauen in sicherheitswahrenden Berufen liegen bei 1,15 Jahren bzw. 2,03 Jahren.“ (BMFSFJ, 2016, 13). 
und für eine Lebensarbeitszeit von 40 Jahren gilt für den geburtsbedingten Teil des BGPG:

$\mathrm{k}^{\circ}=0,40 \cdot 1,3 / 40 \cdot 1,5=1,95 \%{ }^{13}$

Bezieht man diesen Mehrkostensatz von 1,95\% auf einen BGPG von $6 \%$, wie er vom Statistischen Bundesamt ausgewiesen wird, beträgt der Anteil ein Drittel. Bei einer Lebensarbeitszeit von 30 statt 40 Jahren erhöht sich dieser Anteil auf 2,6\% und damit auf annähernd die Hälfte des BGPG.

\section{Zusätzliche Einflussgrößen}

Zu prüfen ist, ob vergleichsweise Mehrkosten durch Krankheit von weiblichen Beschäftigten anfallen. Der Krankenstand von Frauen wäre aber nur dann und in dem Maße zu berücksichtigen, wie er in absoluten Größen und im Vergleich zur mutterschaftsbedingten Abwesenheit signifikant höher als der von Männern läge. Das ist offensichtlich nicht der Fall. ${ }^{14}$ Neben der Krankheit der Beschäftigten selbst erfordert die Krankheit von Kindern die Abwesenheit einer Person vom Arbeitsplatz. Als Lohnersatz wird ein Kinderkrankengeld gezahlt. ${ }^{15}$ Hier kann es über die Lebensarbeitszeit zur mehrfachen Inanspruchnahme von Kinderkrankheitstagen kommen. Sollte der BGPG ausschlaggebend sein, wäre es eher die Frau, die die Betreuung ganz oder überwiegend übernimmt. Wenn allerdings Lohngleichheit hergestellt ist, bleibt aus rein wirtschaftlichen Gründen offen, wer im Fall der Krankheit von Kindern zuhause bleibt, sodass es aus diesem Anlass keinen Grund für eine abwesenheitsbedingte Ungleichbehandlung gibt.

Schließlich ist zu sehen, dass der in dieser Untersuchung zugrunde gelegte BGPG des Statistischen Bundesamts ein - in mehrfacher Hinsicht - Durchschnittswert ist, der z. B. innerhalb und zwischen Branchen ${ }^{16}$, nach Wertigkeit der Arbeit und im Laufe der Lebensarbeitszeit (Beblo und Wolf, 2003) unterschiedlich ausfällt. Je höher z. B. die Position in der Hierarchie des Unternehmens, desto höher auch die (absoluten) Opportunitätskosten der Abwesenheit einer Mutter. ${ }^{17}$ Das Ergebnis könnte dann die Verweigerung einer

13 Bei einem durchschnittlichen Bruttomonatsverdienst für vollzeitbeschäftigte Männer von rund 4.000 Euro (2019) entspricht dem ein absoluter Betrag von rund 80 Euro (Destatis, 2021, c).

14 Nach einer Studie der DAK-Gesundheit lag der Krankenstand der Frauen z.B. 2015 um 14\% höher als derjenige der Männer (DPA, 2016). Demnach fehlten täglich 44 von 1.000 Frauen bei der Arbeit, bei Männern waren es 39 von 1.000 .

15 Vor der Ausnahmesituation der Pandemie standen jedem Elternteil pro Kind maximal zehn Tage Kinderkrankengeld und -tage im Jahr zu.

16 So hat im Architekturbereich der BGPG, der Unterschiede in der Art der Berufsausübung ausschaltet, eine Spannweite von $-2 \%$ bis $+13 \%$ (Reiß \& Hommerich/Bundesarchitektenkammer, 2021, 5).

17 "Furthermore, a quantile decomposition reveals higher wage differentials for outer quantiles and increased importance of the unexplained part for higher quantiles."(Boll und Leppin, 2015).
Beförderung bzw. der Übertragung von Leitungsverantwortung in privaten wie öffentlichen Organisationen sein. In diesem Fall bedarf es der Festlegung einer Quote.

Den Kosten der Diskontinuität durch geburtsbedingte Abwesenheit gegenzurechnen ist eine Bereicherung des Unternehmens durch Diversität, d.h. durch die Zusammenarbeit von Frauen und Männern. So wird vielfach für ein gemischtes Führungsteam plädiert. Manche Unternehmensbeteiligte bestehen sogar darauf (Werner, 2021). Ziel muss aber nicht nur in der Unternehmensführung „eine Hybridkultur sein, die Platz schafft für die Unterschiede und für die Stärken beider Geschlechter." (Heuser und Steinborn, 2013, 63 ff., 123)

Wenn sich keine weiteren „einkommensrelevanten“ und „marktmäßigen“ Merkmale mehr finden lassen, sich die zuvor angesprochenen zusätzlichen Einflussgrößen in etwa die Waage halten und entstandene geburtsbedingte Mehrkosten ausgeglichen werden sollen, müsste im Ergebnis das Unternehmen einen entsprechenden Transfer erhalten und diesen in Form eines höheren Lohns der Frauen weitergeben. Für eine residuale Diskriminierung, die für einen verbleibenden Rest des BGPG verantwortlich sein kann, gibt es dann keinen rechnerischen Grund mehr. Unterbleibt diese, könnte so zwischen den Unternehmen, unabhängig vom Anteil von Frauen und Männern an der Belegschaft, Rentabilitäts- und Wettbewerbsneutralität hergestellt, Frauen bei gleicher Qualifikation der gleiche Lohn wie den Männern gezahlt und damit im privaten Haushalt einkommensmäßig Parität hergestellt werden.

\section{Ansatzpunkte für einen Mehrkostenausgleich}

In der wissenschaftlichen Literatur wird oft politischer Handlungsbedarf angemahnt, um die weitere Entwicklung durch Sozialpartner und/oder Staat in die gewünschte Richtung zu lenken. Nachfolgend werden einige Instrumente angesprochen und miteinander verglichen, die nicht nur für einen Ausgleich geburtsbedingter Mehrkosten $^{18}$, sondern für die Schließung der gesamten bereinigten Lohnlücke diskutiert werden können. ${ }^{19}$ Dabei ist auch eine Kombination verschiedener Instrumente und Finanzierungsquellen möglich.

- Eine obligatorische Lohngleichheit bei sonst gleichen Bedingungen würde das Problem des gleichen Lohns unmittelbar lösen. Offen bliebe aber ein Ausgleich ge-

18 Eine Auszahlung des Ausgleichs könnte mit der Erstattung des anteiligen Mutterschaftsgelds verbunden werden.

19 Jedes dieser Instrumente verdient eine umfassende Darstellung, die hier nicht geleistet werden kann. Wichtig ist indessen, dass ihre Eignung für einen Abbau des BGPG und die Vergleichbarkeit untereinander richtig wiedergegeben werden. 
burtsbedingter Mehrkosten. Wenn dieser Ausgleich entscheidungsrelevant ist, besteht die Gefahr, dass in der Praxis eine obligatorische Lohngleichheit auf Kosten der Beschäftigung von Frauen gehen könnte. Eine Option, dies zu vermeiden, wäre, die Verpflichtung zur Lohngleichheit mit der Erstattung von Mehrkosten zu verbinden.

- Eine Quote richtet sich auf einen höheren Beschäftigungsanteil von Frauen, ohne in jedem Fall Entgeltgleichheit mit zu realisieren.

- Auch Zertifikate können - in flexiblerer Weise - zu einer Mehrbeschäftigung von Frauen beitragen (Akyol, Neugart und Pichler, 2015). Werden die Kosten für die Zertifikate an den Unternehmenssektor zurückgegeben, ist das Instrument für den Sektor insgesamt aufkommensneutral. Lohnparität bei sonst gleichen Bedingungen müsste aber auch hier zusätzlich hergestellt werden.

- Eine Finanzierung aus öffentlichen Mitteln zur Erstattung geburtsbedingter Mehrkosten zielt primär auf Entgeltgleichheit.

- Entgeltgleichheit und eine sektoral aufkommensneutrale Finanzierung ließe sich generell auch dadurch bewirken, dass die Angleichung der Löhne bei sonst gleichen Bedingungen durch die Kombination einer Abgabe des Unternehmens auf den Lohn des Mannes mit einer gleich hohen Subvention des Lohns der Frau (Malus-Bonus-System) hergestellt wird. Wegen der gleichzeitigen Subvention des Lohns der Frau braucht die Höhe der Abgabe bei in etwa gleichen Beschäftigungsanteilen nur die Hälfte der Differenz zwischen Männer- und Frauenlohn zu betragen.

- Ein weiteres Instrument als Beitrag zur Entgeltgleichheit könnte die Erhöhung des Umlagesatzes zur Umlage U2 sein. Ihr Aufkommen knüpft über die Bruttolohn- und -gehaltsumme in etwa an die gleiche Bemessungsgrundlage wie der GPG und der BGPG an.

- Ein eher perspektivisches Instrument jenseits von monetärem Ausgleich wären paritätische Zeitkonten. Im Verlaufe der Lebensarbeitszeit müssten dann Männer wie Frauen idealerweise die gleiche Abwesenheit vom Unternehmen realisieren. Unter dieser Voraussetzung gäbe es für ein rentabilitätsorientiertes Unternehmen keinen Anlass mehr, wegen voraussichtlich unterschiedlicher Abwesenheitszeiten Unterschiede in der Behandlung von Frauen und Männern zu machen. Gleichzeitig gilt dann auch für die Anwesenheit im Haushalt Parität. Einige derzeitige und geplante famili-
Tabelle 1

Ziele, Instrumente und zu erwartende Ergebnisse

\begin{tabular}{|c|c|c|}
\hline Instrument: $\quad$ Unternehmen: & $\begin{array}{l}\text { Beschäftigung von } \\
\text { Frauen }\end{array}$ & Entgeltausgleich \\
\hline \multicolumn{3}{|c|}{ Beschäftigungsrelevante Instrumente } \\
\hline Quote & mehr & nein \\
\hline Zertifikate & mehr & nein \\
\hline \multicolumn{3}{|l|}{ Entgeltrelevante Instrumente } \\
\hline Obligatorische Lohngleichheit & eventuell weniger & ja \\
\hline Öffentliche Finanzierung & offen & ja \\
\hline Abgabe/Subvention & offen & ja \\
\hline Erhöhung der Umlage U2 & offen & ja \\
\hline Paritätische Zeitkonten & offen & ja \\
\hline
\end{tabular}

Quelle: eigener Überblick.

enpolitische Regelungen wie die Partnerschaftsmonate beim Basiselterngeld oder der Partnerschaftsbonus sind erste Schritte hin zu paritätischen Zeitkonten. Ein weitergehender Vorschlag beinhaltet neben dem „Einstieg in den Ausstieg aus dem Ehegattensplitting“ ein „Symmetrisches Elterngeldmodell $3+8+3$ mit mehr exklusiven Monaten und Dynamisierung" (Walper und Beblo, 2021). ${ }^{20}$

Tabelle 1 fasst Ziele, Instrumente und zu erwartende Ergebnisse im Überblick zusammen. Dabei bedeutet das Attribut „offen“, dass in dem Fall, in dem volle oder zumindest annähernde Lohngleichheit erreicht ist, entscheidungsoffen bleibt, wer wie lange Arbeit im Unternehmen oder im Haushalt leistet.

\section{Schlussbemerkung}

Ob sich über die oben ermittelte geburtsbedingte Ursache hinaus weitere einkommensrelevante Gründe für den BGPG finden lassen, muss Gegenstand weiterer Forschung bleiben. Offen bleibt auch, wie stark sich ein Unternehmen bei der Behandlung von Frau und Mann von Rentabilitätsüberlegungen leiten lässt. Ein Ausgleich des aufgezeigten rentabilitätsbezogenen Unterschieds zwischen Mann und Frau könnte jedoch zu gleichen Löhnen unter sonst gleichen Bedingungen und so zu einer Gleichbehandlung von Frauen und Männern sowohl auf Unternehmens- wie Familienebene beitragen.

20 Der Vorschlag entspricht der Praxis in Island; die bezahlte Elternzeit muss dort zu jeweils einem Drittel durch jeden Elternteil genommen werden. Das übrige Drittel kann frei aufgeteilt werden. (Deutscher Bundestag, 2021, 424) 


\section{Literatur}

Akyol, M., M. Neugart und S. Pichler (2015), A tradable employment quota, Beiträge zur Jahrestagung des Vereins für Socialpolitik 2015: Ökonomische Entwicklung - Theorie und Politik - Session: Labor 2, B17-V1.

Arbeitsvertrag.org (2021), https://www.arbeitsvertrag.org/ueberstundenzuschlag/ (15. Juni 2021).

Beblo, M. und E. Wolf (2003), Sind es die Erwerbsunterbrechungen? Ein Erklärungsbeitrag zum Lohnunterschied zwischen Frauen und Männern in Deutschland, Mitteilungen aus der Arbeitsmarkt- und Berufsforschung, 4, 560-572.

Boll, C. und J. Leppin (2015), Die geschlechtsspezifische Lohnlücke in Deutschland: Umfang, Ursachen und Interpretation, Wirtschaftsdienst, 95(4), 249-254, https://www.wirtschaftsdienst.eu/inhalt/jahr/2015/ heft/4/beitrag/die-geschlechtsspezifische-lohnluecke-in-deutschland-umfang-ursachen-und-interpretation.html (15. Juni 2021).

BMFSFJ (Bundesministerium für Familie, Senioren, Frauen und Jugend), (2016), Dauerhaft ungleich - berufsspezifische Lebenserwerbseinkommen von Frauen und Männern in Deutschland, Kurzfassung einer Studie des Hamburgischen WeltWirtschaftsInstituts (HWWI).

BMFSFJ (Bundesministerium für Familie, Senioren, Frauen und Jugend) (2020), Auf dem Weg zur Entgeltgleichheit von Frauen und Männern Daten, Ursachen, Maßnahmen, Stand: Juli 2020.

Destatis (2020,a), Gender Pay Gap 2019: Verdienstunterschied zwischen Männern und Frauen erstmals unter 20\%, Pressemitteilung, 484 vom 8. Dezember.

Destatis (2020, b), Bevölkerung, Geburten.

Destatis (2021, a), Gender Pay Gap 2020 - Frauen verdienen $18 \%$ weniger als Männer, Pressemitteilung, 106 vom 9. März 2021.

Destatis (2021, b), Fachserie 18, Reihe 1.4, Volkswirtschaftliche Gesamtrechnungen, Inlandsproduktberechnung, Detaillierte Jahresergebnisse.

Destatis (2021, c), Verdienste und Verdienstunterschiede, Durchschnittliche Bruttomonatsverdienste, Zeitreihe.

DPA (2016), Arbeit: Studie: Frauen haben mehr Krankheitstage als Männer, Süddeutsche Zeitung, 15. März.

Europäische Kommission (2021), Vorschlag für eine Richtlinie des Europäischen Parlaments und des Rates zur Stärkung der Anwendung des Grundsatzes des gleichen Entgelts für Männer und Frauen bei gleicher oder gleichwertiger Arbeit durch Lohntransparenz und Durchsetzungsmechanismen, COM/2021/93 final.

Finke, C., F. Dumpert und M. Beck (2017), Verdienstunterschiede zwischen Frauen und Männern - Eine Ursachenanalyse auf Grundlage der Verdienststrukturerhebung 2014, Wirtschaft und Statistik, Bd. 2, 43-62.

Heuser, U. J. und D. Steinborn (2013), Anders denken! - Warum die Ökonomie weiblicher wird.

IG Metall (2018), Metall- und Elektroindustrie - IG Metall, https://www.igmetall.de, 5. April - Metall- und Elektroindustrie. Zuschläge für Mehr-, Schicht-, Wechselschicht- und Nachtarbeit.

Institut der deutschen Wirtschaft (IW) (2021), Der Gender Pay Gap in Deutschland und Bayern: Eine gesamtwirtschaftliche Analyse unter besonderer Berücksichtigung des Haushaltskontexts. Eine Untersuchung auf der Basis des Sozio-ökonomischen Panels, Gutachten.

Klammer, U., C. Klenner und S. Lillemeier (2018), „Comparable Worth“ - Arbeitsbewertungen als blinder Fleck in der Ursachenanalyse des Gender Pay Gaps?, Projektbericht, Wirtschafts- und Sozialwissenschaftliches Institut (WSI) in der Hans-Böckler-Stiftung, Study 014, Juni 2018.

Kohlrausch, B. und A. Zucco (2020), Die Corona-Krise trifft Frauen doppelt, Weniger Einkommen und mehr Sorgearbeit, Wirtschafts- und Sozialwissenschaftliches Institut (WSI) in der Hans-Böckler-Stiftung, Policy Brief, 40, 5 .

Reiß \& Hommerich/Bundesarchitektenkammer (2021), Geschlechtsspezifische Gehaltsunterschiede bei angestellten Kammermitgliedern. Eine Sonderauswertung der Daten der bundesweiten Strukturbefragung der Architektenkammern der Länder im Jahr 2020.

Schmidt, J. (2016) Welche Ursachen hat der Gender Pay Gap?, IW-Kurzbericht, 30, 13. Juni.

Schmieder, J. und K. Wrohlich (2021), Gender Pay Gap im europäischen Vergleich: Positiver Zusammenhang zwischen Frauenerwerbsquote und Lohnlücke, Deutsches Institut für Wirtschaftsforschung (DIW), Wochenbericht, 9.

Walper, S. und M. Beblo (2021), Austausch zum Familienbericht am 3. März 2021 auf Einladung des BMFSFJ.

Werner, K. (2021), Geld, Macht und Frauen, Süddeutsche Zeitung, 28. Mai.

Zucco, A. und Y. Lott (2021), Stand der Gleichstellung - Ein Jahr mit Corona, Report, Wirtschafts- und Sozialwissenschaftliches Institut (WSI) in der Hans-Böckler-Stiftung, 64, März. 